\title{
Preliminary Results of the Fermi High-Latitude Extended Source Catalog
}

\author{
Matthew Wood* \\ Kavli Institute for Particle Astrophysics and Cosmology, SLAC National Accelerator Laboratory \\ E-mail: mdwoodeslac.stanford.edu
}

\section{Jonathan Biteau}

Insitut de Physique Nucléaire d'Orsay, Université Paris-Sud, Univ. Paris/Saclay, CNRS/IN2P3,

91400 Orsay, France

E-mail: biteaudipno.in2p3.fr

\section{Regina Caputo}

NASA, GSFC

E-mail: regina.caputo@nasa.gov

\section{Mattia Di Mauro}

Kavli Institute for Particle Astrophysics and Cosmology, SLAC National Accelerator Laboratory

E-mail: mdimauroeslac.stanford.edu

\section{Manuel Meyer}

Kavli Institute for Particle Astrophysics and Cosmology, SLAC National Accelerator Laboratory

E-mail: mameyerestanford.edu

\section{on behalf of the Fermi-LAT Collaboration}

We report on preliminary results from the Fermi High-Latitude Extended Sources Catalog (FHES), a comprehensive search for spatially extended $\gamma$-ray sources at high Galactic latitudes $\left(|b|>5^{\circ}\right)$ based on data from the Fermi Large Area Telescope (LAT). While the majority of high-latitude LAT sources are extragalactic blazars that appear point-like within the LAT angular resolution, there are several physics scenarios that predict the existence of populations of spatially extended sources. If Dark Matter consists of Weakly Interacting Massive Particles, the annihilation or decay of these particles in subhalos of the Milky Way would appear as a population of unassociated $\gamma$-ray sources with finite angular extent. $\gamma$-ray emission from blazars could also be extended (so-called pair halos) due to the deflection of electron-positron pairs in the intergalactic magnetic field (IGMF). The pairs are produced in the absorption of gamma rays in the intergalactic medium and subsequently up-scatter photons of background radiation fields to $\gamma$-ray energies. Measurement of pair halos could provide constraints on the strength and coherence length scale of the IGMF. In a dedicated search, we find 21 extended sources and 16 sources not previously characterized as extended. Limits on the flux of the extended source components are used to derive constraints on the strength of the IGMF using spectral and spatial templates derived from Monte Carlo simulations of electromagnetic cascades. This allows us to constrain the IGMF to be stronger than $3 \times 10^{-16} \mathrm{G}$ for a coherence length $\lambda \gtrsim 10 \mathrm{kpc}$. 


\section{Introduction}

The study of extended $\gamma$-ray sources gives insight into particle acceleration, $\gamma$-ray emission processes, and enables searches for physics beyond the Standard Model. The predominant classes of spatially extended $\gamma$-ray sources in the GeV band are supernova remnants (SNRs) and pulsar wind nebulae (PWNe). In these sources particle acceleration to $\gamma$-ray energies is believed to be driven by shocks in the expanding SNR shell (in the case of SNRs) or at the termination shock of the pulsar wind (in the case of PWNe). A smaller class of extended $\gamma$-ray sources are star-forming regions (SFRs) for which only a few examples are currently known including the Cygnus Cocoon [1] and 30 Doradus region of the LMC [2,3]. Particle acceleration in these objects may be driven by some combination of stellar winds or successive supernova explosions. Extended sources are also found associated with the diffuse emission from nearby galaxies: M31 [4], Fornax A [5], LMC [2], SMC [6], and Cen A [7].

Beyond the known classes of extended $\gamma$-ray sources, there are several exotic scenarios that predict populations of extragalactic extended sources. If Dark Matter (DM) consists of Weakly Interacting Massive Particles (WIMPs), the annihilation or decay of DM in subhalos of the Milky Way could result in a population of spatially extended, unassociated, $\gamma$-ray sources (e.g. [8]). Extended emission could also be associated with the electromagnetic cascades generated by VHE gamma rays emitted by $\gamma$-ray blazars. The $e^{+} e^{-}$pairs produced when VHE photons undergo pair production can in turn inverse-Compton (IC) scatter photons of the cosmic microwave background (CMB), thereby initiating the cascade [9]. The pairs are deflected in the intergalactic magnetic field (IGMF) and, depending on its strength and coherence length, an extended $\gamma$-ray halo can form around the AGN, often referred to as a pair halo [10]. Measurement of pair halos could be used to constrain the properties of the IGMF.

We report here on preliminary results of the Fermi High-Latitude Extended Sources Catalog (FHES), a comprehensive search for spatially extended $\gamma$-ray sources above $5^{\circ}$ Galactic latitude using 7.5 years of Pass 8 data above $1 \mathrm{GeV}$. We further examine the spatial and spectral properties of the TeV blazars in our sample to look for evidence of pair halos and derive constraints on the IGMF.

\section{Data Analysis}

We analyze 90 months of LAT data (2008 August 4 to 2016 February 4) selecting P8R2 SOURCE-class events in the energy range from $1 \mathrm{GeV}$ to $1 \mathrm{TeV}$. We perform a binned maximumlikelihood analysis with eight logarithmic bins per decade in energy and a region of interest (ROI) of $6^{\circ} \times 6^{\circ}$ with an angular pixelization of 0.025 . To maximize the sensitivity to small angular extensions, we split the data into two independent samples according to the quality of the angular reconstruction: evtype=32 (PSF3) and evtype=28 (PSF0+PSF1+PSF2), where PSF stands for point spread function.

We consider 2693 regions of interest (ROIs) centered on sources listed in the 3FGL and 3FHL catalogs $[11,12]$ with $|b|>5^{\circ}$. We use the Galactic Interstellar Emission Model (IEM) recommended for Pass 8 analysis (gll_iem_v06.fits) and standard templates for the isotropic

\footnotetext{
* Speaker.
} 
background. We include in our model 3FGL sources within a region of $10^{\circ} \times 10^{\circ}$ centered on each ROI. We exclude 3FGL sources detected with a Test Statistic (TS) ${ }^{1}$ less than 100 or analysis flags indicating confusion with diffuse emission (flags 5,6 , or 8 ).

We optimize each ROI by first re-fitting the spectral parameters of all model components and the positions of point sources inside the ROI boundary. We then identify new point source candidates in two passes. We first identify new point sources in the outer ROI $\left(R>1.0^{\circ}\right)$ with TS $>9$ and fit the spectrum and position for each. We then iteratively search for new point sources in the inner ROI while testing the central source for extension. With each additional point source we test the hypothesis of $n$ point sources against two alternative hypotheses: substituting the central source with a 2D Gaussian with the same spectral parameterization (extension hypothesis) and superimposing the central source with a 2D Gaussian at the same position with a power-law spectrum (halo hypothesis). We stop adding new point sources when no additional candidates are found or when a model of extension is found to be preferred according to the Akaike information criterion (AIC, see Ref. [13]) given by AIC $=2 k-2 \ln \mathscr{L}$ where $k$ is the number of parameters in the model.

After selecting the best-fit iteration $n$, we evaluate the statistical evidence for extended emission from the likelihood ratio of models with and without an extended component,

$$
\mathrm{TS}_{\mathrm{m}}=2\left(\ln \mathscr{L}_{\mathrm{n}+\mathrm{m}}-\ln \mathscr{L}_{\mathrm{n}}\right),
$$

where $\mathrm{m}=$ ext (halo) for the extension (halo) hypothesis, $\mathscr{L}_{\mathrm{n}}$ is the likelihood for the model with $n$ point sources, and $\mathscr{L}_{\mathrm{n}+\mathrm{m}}$ is the likelihood for the halo or extension hypothesis. We identify a source as extended if $\mathrm{TS}_{\mathrm{ext}}>16$ or $\mathrm{TS}_{\text {halo }}>16$ where in the latter case we reanalyze the source after adding an extended component with the spatial and spectral parameters of the best-fit halo.

\section{Extended Source Catalog}

Our analysis detects 21 sources with statistically significant evidence for extension $\left(\mathrm{TS}_{\mathrm{ext}}>\right.$ 16). Figure 1 shows the the distribution of the new extended source candidates. Of these extended sources, 5 were previously detected as extended and 16 are new. Of the new sources, 8 have potential associations including two sources associated to Cen A, two SNRs, and an extended source consistent with the position of the Crab Nebula. However, the significance of the extension of this source drops significantly and is below $\mathrm{TS}_{\mathrm{ext}}=16$ if systematic uncertainties on the PSF are considered. The remaining eight unassociated sources all have $|b|<20^{\circ}$ consistent with a Galactic origin.

We identify three sources that we tentatively associate to SFRs by their spatial correlation with multiwavelength tracers of star-formation activity. All three sources are found to be robust to IEM systematics and have no apparent correlation to features in the gas or dust maps of the respective regions. The left panel of Figure 2 shows the residual TS map for FHES J0000.9+6831, an extended FHES sources that we associate to the star-forming HII region NGC 7822. FHES J0000.9+6831 has an extension of $0.95^{\circ}$ and a soft- $\gamma$-ray spectrum $(\Gamma \simeq 2.7)$ that is consistent with the typical spectrum arising from CR interactions with the ISM. NGC 7822 is part of the Cepheus OB4 starforming region which is located at a distance of $1 \mathrm{kpc}$. An overdensity of $\mathrm{O}$ and $\mathrm{B}$ stars is observed

\footnotetext{
${ }^{1} \mathrm{TS}$ is defined as twice the log likelihood ratio between models with and without the source.
} 


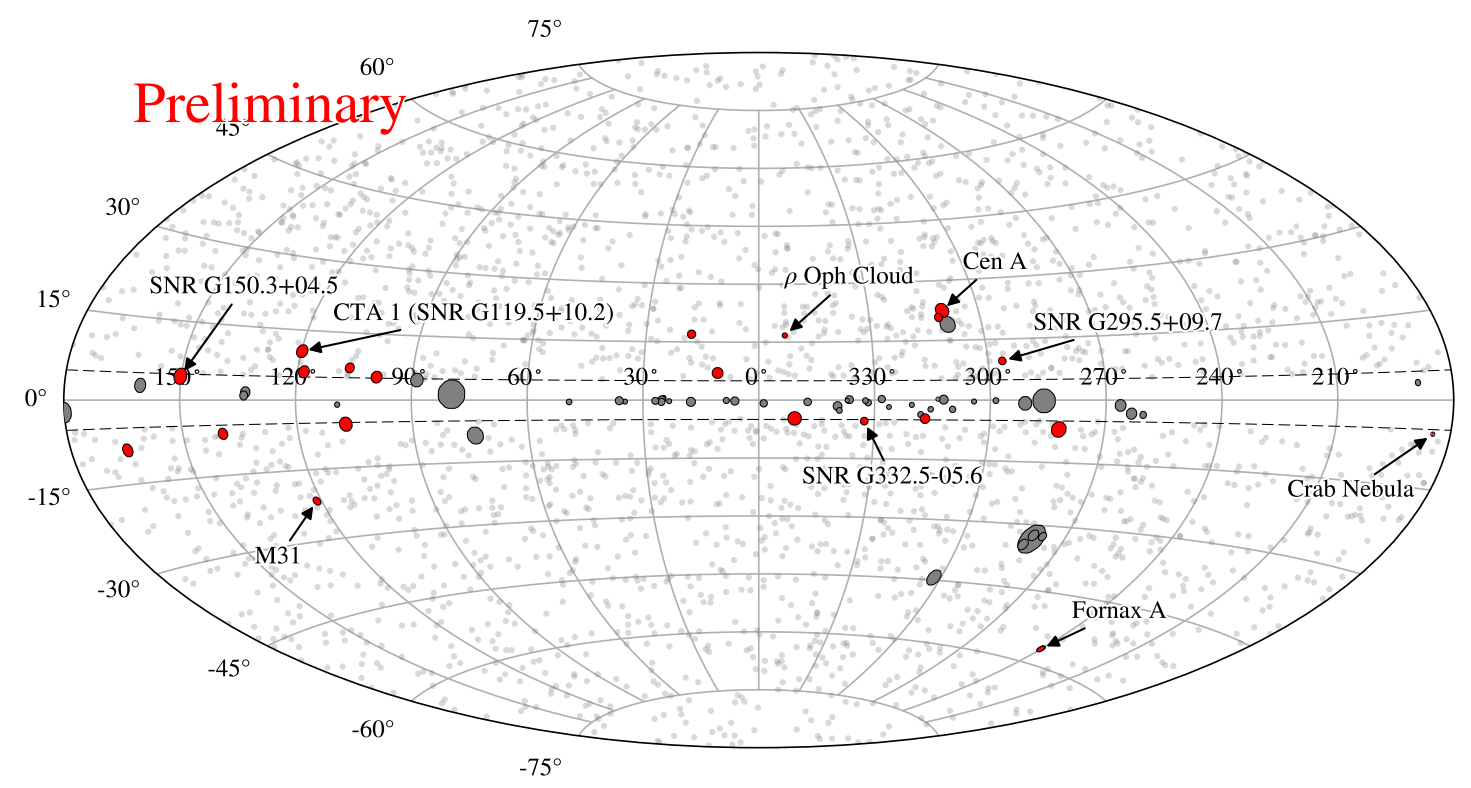

Figure 1: Distribution of FHES sources in Galactic coordinates. Light grey markers indicate sources that are best fit by a point-source morphology $\left(\mathrm{TS}_{\mathrm{ext}}<16\right)$. Red circles with black outline indicate FHES sources that are best fit by an extended morphology $\left(\mathrm{TS}_{\text {ext }}>16\right)$. The size of the marker is drawn to scale of the intrinsic $68 \%$ containment radius of the source. Labeled sources are those with a previously published detection of extension or an unambiguous association to a multiwavelength counterpart. Grey circles with black outline indicate the position and angular size of known LAT extended sources that fell outside our latitude selection or were explicitly excluded from the analysis.

within the region encompassed by FHES J0000.9+6831 indicating that the $\gamma$-ray source may be associated with the collision of stellar winds driven by O/B stars. A correlation is also observed with a shell-like feature in the map of emission at $22 \mu \mathrm{m}$ from WISE which traces the thermal emission of dust heated by young, massive stars in the region.

An example of a source without clear association is FHES J1741.5-3920, shown in the right panel of Figure 2. It has the largest angular size $\left(D \sim 3.0^{\circ}\right)$ and hardest $\gamma$-ray spectrum $(\Gamma=$ $1.8 \pm 0.04)$ of the unassociated FHES sources. We note that [14] report the discovery of a new $\gamma$-ray source with the same position, morphology, and spectral characteristics. The spectrum and morphology of this source suggest that it may be associated with a young shell-type remnant. FHES J1741.5-3920 is positionally coincident with SNR G351.0-5.4 [15], a radio-detected SNR with $D \sim 0.5^{\circ}$. However, an association with this SNR is disfavored given the large mismatch in angular size.

\section{IGMF Limits}

We do not find evidence for an extension of blazars and use the FHES to derive constraints on the coherence length, $\lambda$, and field strength, $B$, of the IGMF. We use both spectral and spatial information of the catalog as well as spectra from imaging air Cherenkov telescopes (IACTs) to 

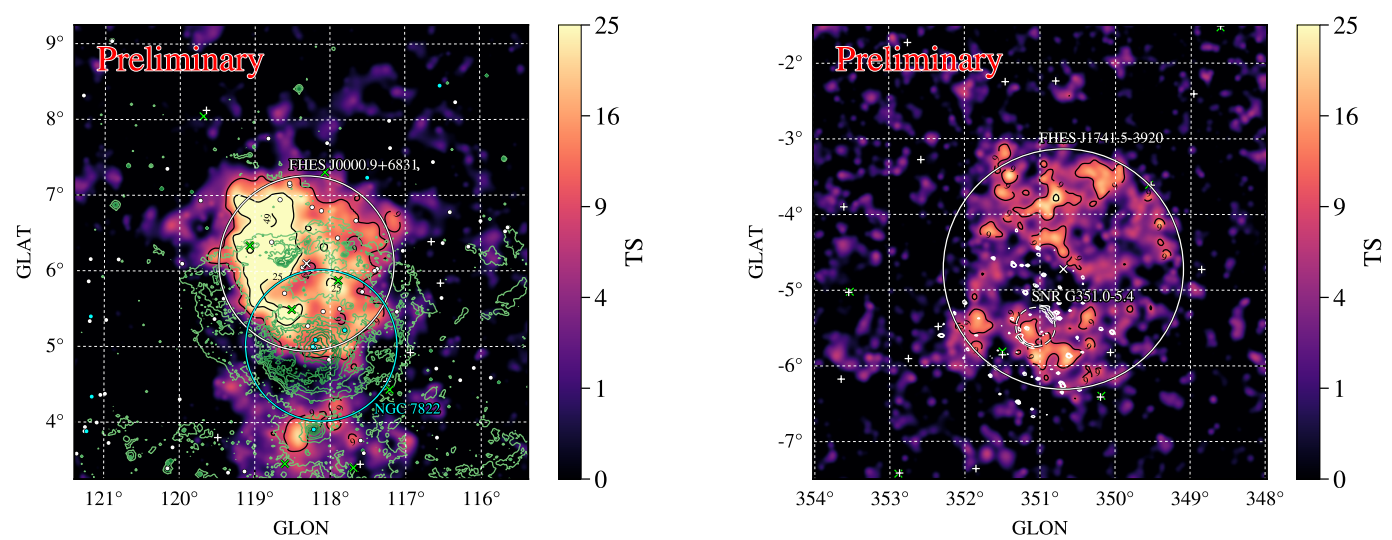

Figure 2: Residual TS maps for the FHES extended source candidates FHES J0000.9+6831 (left) and FHES J1741.5-3920 (right). The white circle with central cross indicates the best-fit disk extension and centroid of the FHES source. White pluses indicate point-source candidates from the best-fit model for the region. Green crosses indicate the positions of sources in the 3FGL catalog. White and cyans circles indicate the locations of $\mathrm{O}$ and $\mathrm{B}$ stars from the SIMBAD database. Green contours in the left plot show the WISE map of $22 \mu \mathrm{m}$ emission. The cyan circle indicates the extent of the HII region NGC 7822 which is tentatively associated to FHES J0000.9+6831. White contours and white dashed circle in the right panel show the GMRT radio map of SNR G351.0-5.4 at $325 \mathrm{MHz}$ [15] and its inferred position and diameter.

derive these constraints. For this study we select 9 BL Lac objects with known redshifts and wellmeasured $\mathrm{TeV}$ spectra.

We use the ELMAG Monte Carlo code [16] to generate models for the cascade component associated with each object in our sample. This code computes the expected flux and angular distribution of the cascade by propagating individual $\gamma$-rays and modeling the electromagnetic cascades produced when these photons pair convert on CMB and EBL photons. ELMAG uses a simplified model of the IGMF that splits the field into cells with constant field strength and size equal to the coherence length scale. In addition to the IGMF parameters, the cascade depends on the intrinsic properties of the blazar: the redshift $(z)$, the spectrum $(\phi(\mathbf{p}))$ with intrinsic parameters $\mathbf{p}$, the jet opening angle $\left(\theta_{\text {jet }}\right)$, and the source activity time scale $\left(t_{\max }\right)$. We simulate the full cascade spectrum over a grid of redshifts and in bins of injected $\gamma$-ray energy $\Delta E$ between $100 \mathrm{MeV}$ and $32 \mathrm{TeV}$ (using again 8 bins per decade) using the EBL model of [17]. Using these simulations we compute a grid of models for the flux and angular size of the cascade as a function of the IGMF and source parameters. We derive limits on the IGMF by performing a joint fit to the LAT and IACT measurements of each blazar. We find the best-fit IGMF parameters while simultaneously fitting the parameters of the intrinsic spectrum (p).

The source parameters $\theta_{\text {jet }}$ and $t_{\max }$ are poorly constrained for the sources under consideration. For $\theta_{\text {jet }}$ we choose $6^{\circ}$ which is consistent with the typical blazar jet angles measured from radio observations. For $t_{\max }$ we consider timescales $t_{\max }=10,10^{4}, 10^{7}$ years where $t_{\max }=10 \mathrm{yr}$ is chosen to match the length of the LAT observations and $t_{\max }=10^{7} \mathrm{yr}$ is the expected activity timescale of AGN [18].

The left panel of Figure 3 shows the best-fit spectrum and cascade contribution versus magnetic field strength for one of the objects in our samples, 1ES 1101-232. The right panel of Figure 3 

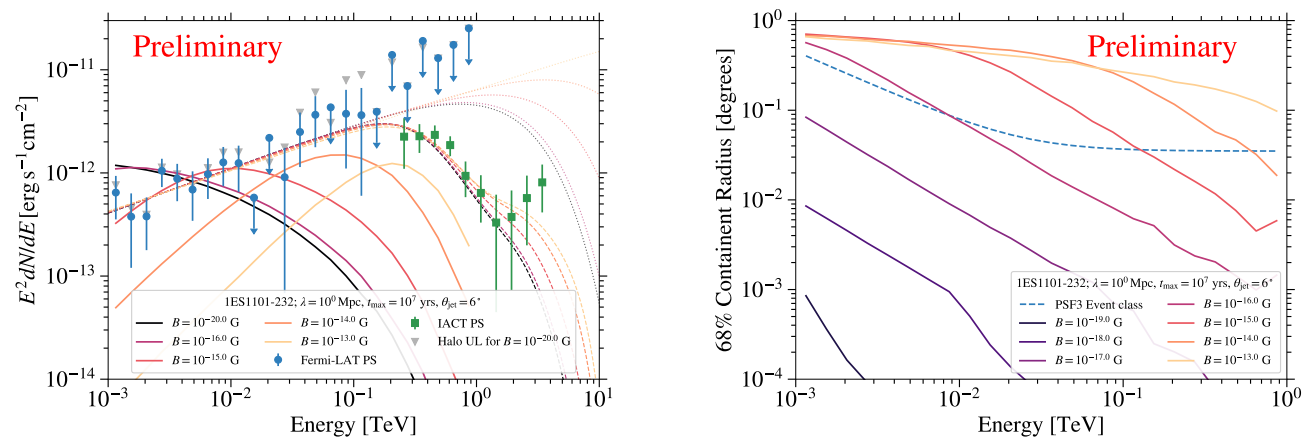

Figure 3: Left: Fit of the intrinsic spectrum and cascade component to the IACT and Fermi-LAT data of 1ES 1101-232 $(z=0.186)$ for different IGMF strengths. A source activity time of $t_{\max }=10^{7}$ years and a jet opening angle of $\theta_{\text {jet }}=6^{\circ}$ are assumed together with a coherence length of $1 \mathrm{Mpc}$. The best-fit intrinsic spectra multiplied with EBL absorption are shown as dashed lines with colors matching those of the cascade component (solid lines). The intrinsic spectra are shown as dotted lines. Upper limits on the halo energy flux for widths equal to that of the cascade for $B=10^{-20} \mathrm{G}$ are shown as gray triangles. Right: Containment radii for the cascade for different $B$-field strengths and the PSF (PSF3 event type) as a function of energy for the same source and parameters as the right panel.

shows the angular size of the cascade as a function of energy and magnetic field strength. In the limit of small magnetic field strengths the cascade component peaks at $\mathrm{GeV}$ energies and has an angular size well below the LAT angular resolution. For larger field strengths the peak of the cascade moves to higher energies. The angular extent of the halo is measurable when $B \gtrsim 10^{-16} \mathrm{G}$.

We find in all cases that the best-fit model is one with a large $B$-field that suppresses the cascade flux to a level below what would be detectable in the LAT energy regime. We obtain $95 \%$ limits in the IGMF parameter space by finding the values of $(B, \lambda)$ that change $2 \ln \mathscr{L}$ by 5.99 corresponding to a $\chi^{2}$ distribution with two degrees of freedom. Figure 4 shows the IGMF limits obtained for individual blazars as well as combined limits from stacking the likelihoods of all of the BL Lac objects in the IGMF sample. Under the short activity timescale scenario $\left(t_{\max }=10 \mathrm{yr}\right)$, we are able to constrain $B \gtrsim 3 \times 10^{-16} \mathrm{G}$ for $\lambda>10^{-2} \mathrm{Mpc}$. For the long timescale scenario $\left(t_{\max }=10^{7} \mathrm{yr}\right.$ ), we can constrain $B \gtrsim 3 \times 10^{-13} \mathrm{G}$ for $\lambda>10^{-2} \mathrm{Mpc}$.

\section{Conclusions}

We report on preliminary results of a search for extended sources at high Galactic latitude. We are able to identify 21 extended sources, 16 of which are identified as such for the first time. All of the unassociated sources are found with $|b|<20^{\circ}$ indicating that they have either a Galactic origin or arise from systematic uncertainties in the IEM. We do not find evidence for extended emission associated with extragalactic source populations either from blazars or unassociated sources.

Using the results of the extended source catalog, we are able to derive constraints on the IGMF that are substantially stronger than previous works using LAT observations of TeV blazars (see e.g. [19]), limiting $B \gtrsim 3 \times 10^{-16} \mathrm{G}$ for $\lambda \gtrsim 10 \mathrm{kpc}$ for an activity time of the considered blazars of 10 years. Using instead activity times of $10^{4}\left(10^{7}\right)$ years improves the limits to $B \gtrsim 9 \times 10^{-15} \mathrm{G}$ 

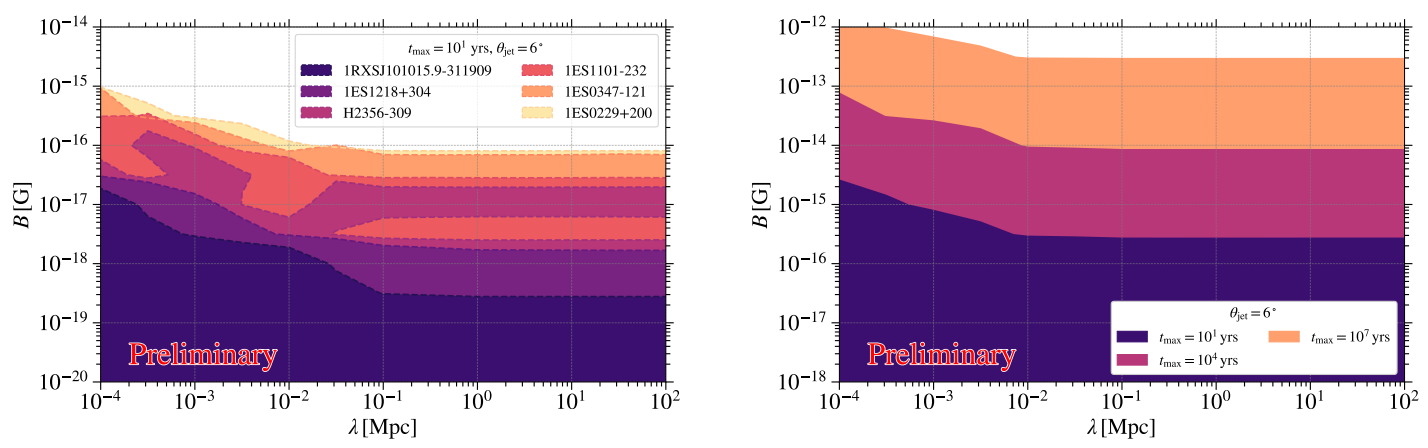

Figure 4: $95 \%$ lower limits on the field strength of the IGMF for $\theta_{\text {jet }}=6^{\circ}$. Left: Exclusions for $t_{\max }=$ 10 years for individual sources. Right: Combined exclusion limits for different blazar activity times.

$\left(B \gtrsim 3 \times 10^{-13} \mathrm{G}\right)$. For such large fields, however, the actual jet opening and viewing angle of the blazar become important to accurately model the halo. The influence of these effects in the limit of large field strength $\left(B \gtrsim 10^{-15} \mathrm{G}\right)$ is not considered in the simplified 1D Monte-Carlo calculation used by ELMAG.

Dedicated 3D Monte Carlo codes should be used in the future to search for the cascade emission at higher values of the IGMF to accurately model the source extension and taking into account the viewing angle of the blazar [20,21,22]. Further extensions could include more realistic models of the intergalactic field, including a full treatment of its turbulence spectrum [23] and its helicity [24].

\section{Acknowledgments}

The Fermi-LAT Collaboration acknowledges support for LAT development, operation and data analysis from NASA and DOE (United States), CEA/Irfu and IN2P3/CNRS (France), ASI and INFN (Italy), MEXT, KEK, and JAXA (Japan), and the K.A. Wallenberg Foundation, the Swedish Research Council and the National Space Board (Sweden). Science analysis support in the operations phase from INAF (Italy) and CNES (France) is also gratefully acknowledged.

\section{References}

[1] M. Ackermann et al., A Cocoon of Freshly Accelerated Cosmic Rays Detected by Fermi in the Cygnus Superbubble, Science 334 (2011) 1103.

[2] M. Ackermann et al., Deep view of the Large Magellanic Cloud with six years of Fermi-LAT observations, Astron. Astrophys. 586 (2016) A71, [1509.06903].

[3] A. Abramowski et al., The exceptionally powerful TeV $\gamma$-ray emitters in the Large Magellanic Cloud, Science 347 (2015) 406-412, [1501.06578].

[4] M. Ackermann et al., Observations of M31 and M33 with the Fermi Large Area Telescope: A Galactic Center Excess in Andromeda?, Astrophys. J. 836 (2017) 208, [1702 . 08602].

[5] M. Ackermann et al., Fermi Large Area Telescope Detection of Extended Gamma-Ray Emission from the Radio Galaxy Fornax A, Astrophys. J. 826 (2016) 1, [1606.04905]. 
[6] Detection of the Small Magellanic Cloud in gamma-rays with Fermi/LAT, Astron. Astrophys. 523 (2010) A46, [1 008 .2127].

[7] A. A. Abdo et al., Fermi Gamma-Ray Imaging of a Radio Galaxy, Science 328 (2010) 725, [1006.3986].

[8] M. Ackermann et al., Search for Dark Matter Satellites using the FERMI-LAT, Astrophys. J. 747 (2012) 121, [1201.2691].

[9] R. J. Protheroe and T. Stanev, Electron-Photon Cascading of Very High-Energy Gamma-Rays in the Infrared Background, MNRAS 264 (1993) 191-+.

[10] F. A. Aharonian, P. S. Coppi and H. J. Voelk, Very high energy gamma rays from active galactic nuclei: Cascading on the cosmic background radiation fields and the formation of pair halos, ApJ 423 (1994) L5-L8, [arXiv:astro-ph/9312045].

[11] F. Acero et al., Fermi Large Area Telescope Third Source Catalog, ApJS 218 (2015) 23, [1501.02003].

[12] The Fermi-LAT Collaboration, 3FHL: The Third Catalog of Hard Fermi-LAT Sources, ArXiv e-prints (2017), [1702.00664].

[13] H. Akaike, A new look at the statistical model identification, IEEE Transactions on Automatic Control 19 (1974) 716-723.

[14] M. Araya, Discovery of an Extended Source of Gamma-ray Emission in the Southern Hemisphere, ArXiv e-prints (2016), [1609.06225].

[15] F. de Gasperin et al., Discovery of the supernova remnant G351.0-5.4, Astron. Astrophys. 568 (2014) A107, [1408.0036].

[16] M. Kachelrieß, S. Ostapchenko and R. Tomàs, ELMAG: A Monte Carlo simulation of electromagnetic cascades on the extragalactic background light and in magnetic fields, Computer Physics Communications 183 (2012) 1036-1043, [1106.5508].

[17] A. Dominguez et al., Extragalactic Background Light Inferred from AEGIS Galaxy SED-type Fractions, Mon. Not. Roy. Astron. Soc. 410 (2011) 2556, [1 007 . 145 9].

[18] P. Parma, M. Murgia, H. R. de Ruiter and R. Fanti, The lives of FR I radio galaxies, New A Rev. 46 (2002) 313-325, [astro-ph/9910254].

[19] J. D. Finke, L. C. Reyes, M. Georganopoulos, K. Reynolds, M. Ajello, S. J. Fegan et al., Constraints on the Intergalactic Magnetic Field with Gamma-Ray Observations of Blazars, ApJ 814 (2015) 20, [1510.02485].

[20] A. Neronov, D. Semikoz, M. Kachelriess, S. Ostapchenko and A. Elyiv, Degree-scale GeV “Jets” from Active and Dead TeV Blazars, ApJ 719 (2010) L130-L133, [1 002 . 4981].

[21] R. Alves Batista, A. Saveliev, G. Sigl and T. Vachaspati, Probing intergalactic magnetic fields with simulations of electromagnetic cascades, Phys. Rev. D 94 (2016) 083005, [1607 . 00320 ].

[22] T. Fitoussi et al., Physics of cosmological cascades and observable properties, MNRAS 466 (2017) 3472-3487, [1701.00654].

[23] C. Caprini and S. Gabici, Gamma-ray observations of blazars and the intergalactic magnetic field spectrum, Phys. Rev. D 91 (2015) 123514, [1504.00383].

[24] W. Chen, B. D. Chowdhury, F. Ferrer, H. Tashiro and T. Vachaspati, Intergalactic magnetic field spectra from diffuse gamma-rays, MNRAS 450 (2015) 3371-3380, [1412 . 3171]. 